\title{
Factors affecting the accuracy of chest compression depth estimation
}

\author{
Jung Hee Kang, Won Chul Cha, Minjung Kathy Chae, Hang A Park, \\ Sung Yeon Hwang, Sang Chan Jin, Tae Rim Lee, Tae Gun Shin, \\ Min Seob Sim, lk Joon Jo, Keun Jeong Song, Joong Eui Rhee, \\ Yeon Kwon Jeong
}

Department of Emergency Medicine, Samsung Medical Center, Sungkyunkwan University School of Medicine, Seoul, Korea

Objective We aimed to estimate the accuracy of visual estimation of chest compression depth and identify potential factors affecting accuracy.

Methods This simulation study used a basic life support mannequin, the Ambu man. We recorded chest compression with 7 different depths from 1 to $7 \mathrm{~cm}$. Each video clip was recorded for a cycle of compression. Three different viewpoints were used to record the video. After filming, 25 clips were randomly selected. Health care providers in an emergency department were asked to estimate the depth of compressions while watching the selected video clips. Examiner determinants such as experience and cardiopulmonary resuscitation training and environment determinants such as the location of the camera (examiner) were collected and analyzed. An estimated depth was considered correct if it was consistent with the one recorded. A multivariate analysis predicting the accuracy of compression depth estimation was performed.

Results Overall, 103 subjects were enrolled in the study; 42 (40.8\%) were physicians, 56 (54.4\%) nurses, and 5 (4.8\%) emergency medical technicians. The mean accuracy was 0.89 (standard deviation, 0.76). Among examiner determinants, only subjects' occupation and clinical experience showed significant association with outcome $(P=0.03$ and $P=0.08$, respectively). All environmental determinants showed significant association with the outcome (all $P<0.001$ ). Multivariate analysis showed that accuracy rate was significantly associated with occupation, camera position, and compression depth.

Conclusions The accuracy rate of chest compression depth estimation was 0.89 and was significantly related with examiner's occupation, camera view position, and compression depth.

Keywords Cardiopulmonary resuscitation; Feedback; Manikins; Patient care team; Video recording

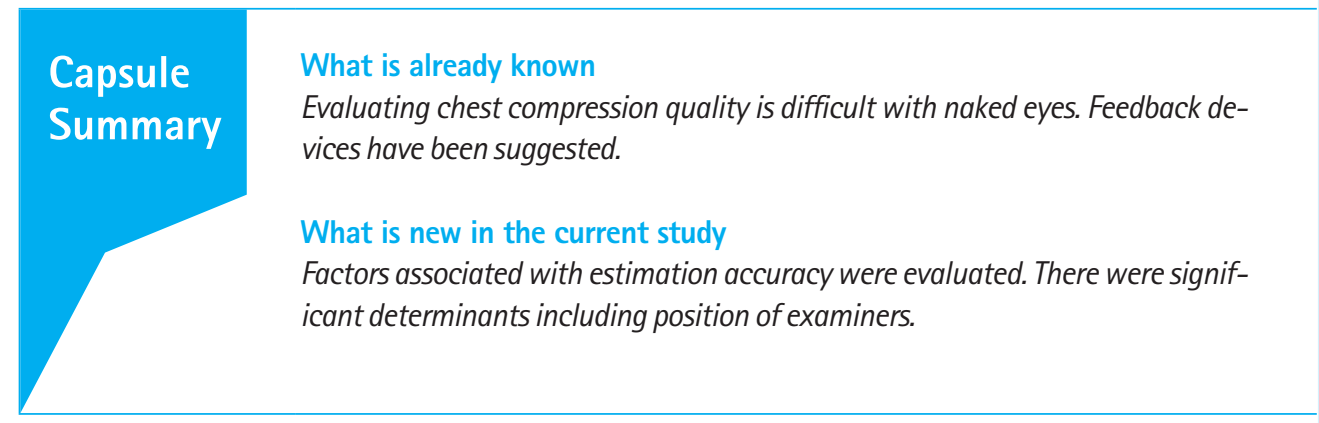

elSSN: 2383-4625

Received: 24 June 2014

Revised: 25 July 2014

Accepted: 29 July 2014

Correspondence to: Won Chul Cha Department of Emergency Medicine, Samsung Medical Center, Sungkyunkwan University School of Medicine, 81 Irwon-ro, Gangnam-gu, Seoul 135-710, Korea

E-mail: docchaster@gmail.com

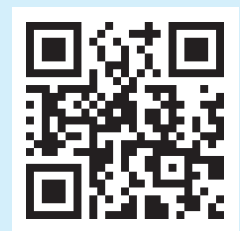

How to cite this article:

Kang $\mathrm{HH}$, Cha WC, Chae MK, Park HA, Hwang SY, Jin SC, Lee TR, Shin TG, Sim MS, Jo IJ, Song KJ, Rhee JE, Jeong YK. Factors affecting the accuracy of chest compression depth estimation. Clin Exp Emerg Med 2014;1(2):101-108.

This is an Open Access article distributed under the terms of the Creative Commons Attribution Non-Commercial License (http:// creativecommons.org/licenses/by-nc/3.0/). 


\section{INTRODUCTION}

High-quality chest compression is an essential element for achieving a good prognosis in cardiac arrest patients. These compressions are composed of an appropriate compression rate ( $>100$ ) $\mathrm{min})$, appropriate chest compression depth $(>5 \mathrm{~cm})$, and sufficient chest recoil. ${ }^{1,2}$ Guidelines state the need for mutual feedback among resuscitation providers to maintain these quality indices. ${ }^{3}$ Based on the 2010 European Resuscitation Council (ERC) guideline, a team leader should evaluate the quality of the cardiopulmonary resuscitation (CPR) and can change the person providing CPR if necessary. ${ }^{4}$

There have been many studies suggesting the effectiveness of feedback devices that measure the rate, depth, and release of chest compression. ${ }^{5-8}$ Information on feedback devices was also included in the American Heart Association (AHA; class lla, level of evidence $B$ ) and ERC guidelines since 2010.,

However, feedback devices are not used frequently in many real-life CPR locations. ${ }^{9}$ Frequently, providers choose not to apply the device because of reluctance and ignorance of effectiveness and time and cost to import new systems. ${ }^{9,10}$ In many cases, feedback is based on visual estimation by the naked eye. Although objective indicators such as compression rate, respiration rate, and end-tidal carbon dioxide concentration are easy to estimate, compression depth can be difficult.

The purpose of our study was to estimate the accuracy of compression depth estimation and to identify potential factors affecting accuracy.

\section{METHODS}

\section{Study design and setting}

This study was approved by the Institutional Review Board (approval number SMC 2014-03-099-001). This study was a simulation study using mannequins (Ambu man, Ballerup, Denmark). Five basic life support providers volunteered to have video clips recorded while they did chest compressions. They were asked to perform chest compressions of different depths: $\geq 0 \&<1, \geq 1 \&$ $<2, \geq 2 \&<3, \geq 3 \&<4, \geq 4 \&<5, \geq 5 \&<6$, and $\geq 6 \&<7 \mathrm{~cm}$. Compressors did chest compression for a cycle of 30 compressions on their knees. To maintain the same level of depth throughout a cycle, a quality manager monitored compression depths using the Smartman, a PC program provided by the manufacturer (Fig. 1D).

Video clips were recorded by a smartphone camera (Galaxy S3, 1080p Recording System, Samsung, Suwon, Korea). We used 3 recording positions: cephalic (Fig. 1A), side (Fig. 1B), and caudal
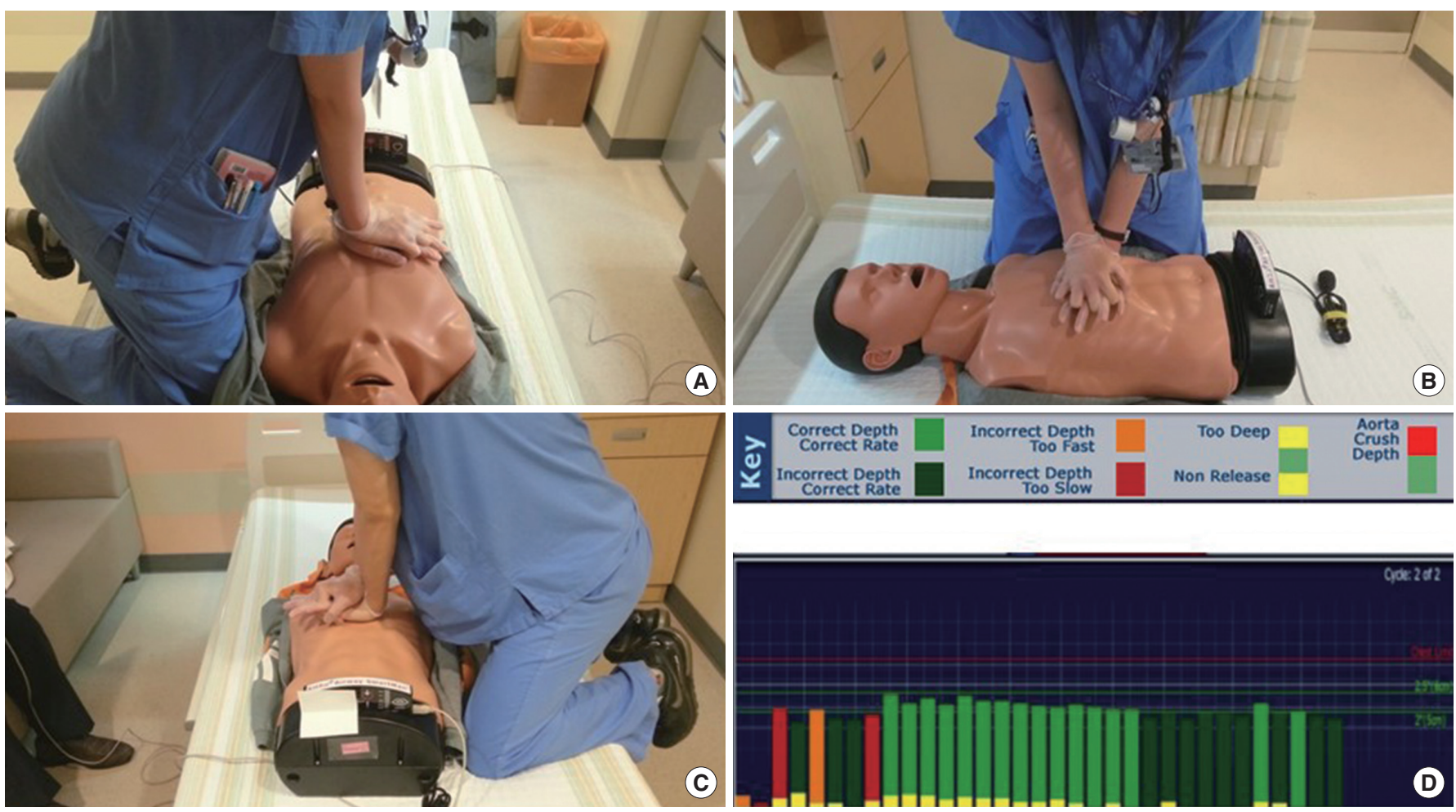

Fig. 1. The process of how video clips were recorded. (A) Cephalic view, (B) side view, and (C) caudal view. (D) The process showing how compression quality and depth are controlled by the computer system, Ambu Man Compression. 
(Fig. 1C). Camera height was fixed at $155 \mathrm{~cm}$ from the floor and $125 \mathrm{~cm}$ from the bed on which the mannequin was placed. Of 175 total clips, we randomly sampled 25, 5 clips from each volunteer. An internet-based randomization program was used for the sampling process (http://randomization.com).

\section{Study participants}

Study participants were enrolled from a single, tertiary, teaching hospital. All were working or have worked in the emergency department. Shortly after explaining the study, participants filled out survey forms and watched the video clips while estimating the depth of each clip.

\section{Methods and measurements}

To measure examiner determinants, we collected information regarding gender, age, occupation, affiliated department, clinical career, resuscitation certification state, and the number of CPR experiences during the past year. To measure the environmental determinants, we included the recording position, chest compressor's gender, and compression depth.

\section{Outcomes}

An estimated depth was considered correct if the recorded depth and the estimated depth were all $>5 \mathrm{~cm}$, or all $<5 \mathrm{~cm}$ (Fig. 2A). For example, if a recorded depth was $1-2 \mathrm{~cm}$ and the answer was $4 \mathrm{~cm}$, the answer was considered correct because both depths were consistent with insufficient compression. We used this criterion because the current AHA guideline states that a provider has to compress $>5 \mathrm{~cm}$.

\section{Sensitivity analysis}

For sensitivity analysis, 2 more criteria were applied. The first criterion was according to the 2010 ERC guideline for resuscitation (Fig. 2B). Our second criterion was a strict one: that estimation was considered correct when the recorded and estimated depths were exactly the same (Fig. 2C).

\section{Analysis}

All statistical analyses were performed with STATA ver. 13.0 (Stata Co., College Station, TX, USA). Baseline characteristics of this study were expressed as numbers, percentages, and means with standard deviations (SDs). The results are presented as mean with SD and 95\% confidence intervals (Cls). Comparisons of continuous data were performed using the t-test and analysis of variance. To identify factors affecting accuracy of compression depth estimation, a multivariate logistic regression was used. P-values $<0.05$ were considered statistically significant for all statistical testing.
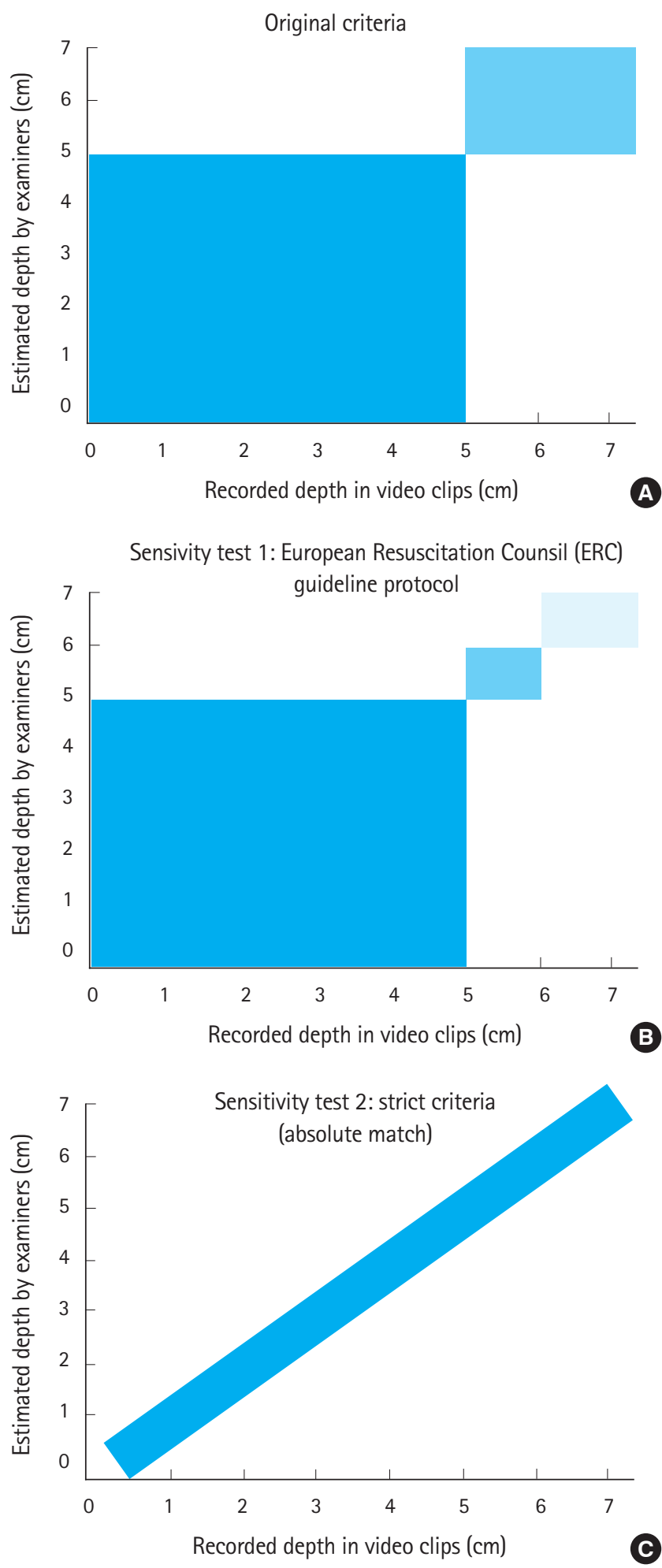

Fig. 2. The shaded areas represent areas where answers were checked as correct (or consistent). (A) Based on the American Heart Association guideline $(>5 \mathrm{~cm}$ ). (B) Based on the European Resuscitation Council guideline 5-6 cm. (C) Exact match. Criteria for (B) and (C) were used for sensitivity analysis. 


\section{RESULTS}

\section{Characteristics of study subjects}

A total of 103 health care providers participated in this study. Table 1 shows the demographic characteristics of the participants. The number of males was 28 (27.2\%). The mean age was 29.5 years (SD, 5.2). Forty-two (40.8\%) were physicians, 56 (54.4\%) were nurses, and 5 (4.8\%) were emergency medical technicians (EMTs); 81 (78.6\%) were currently affiliated with the emergency department; 13 (12.6\%) had valid CPR instructor certification issued by the AHA; and 23 (22.3\%) had advanced cardiac life support certification. In terms of CPR experience, 45 participants (43.7\%) had more than 21 CPR experiences during the past year (Table 1).

\section{Main results}

The mean estimated accuracy was 0.89 (SD 0.76). Table 2 shows the examiner determinants. There was no significant association between accuracy and gender, age, affiliated department, clinical career, instructor certification, or advanced cardiac life support certification. The accuracy rate and number of CPR experiences showed a trend of negative association $(P=0.08)$. The estimated

Table 1. Baseline characteristics of examiners $(n=103)$

\begin{tabular}{|c|c|}
\hline Characteristic & Value \\
\hline \multicolumn{2}{|l|}{ Gender } \\
\hline Male & $28(27.2)$ \\
\hline Female & 75 (72.8) \\
\hline Age (yr) & $29.5 \pm 5.2$ \\
\hline \multicolumn{2}{|l|}{ Occupation } \\
\hline Physician & $42(40.8)$ \\
\hline Nurse & $56(54.4)$ \\
\hline Emergency medical technician & $5(4.9)$ \\
\hline \multicolumn{2}{|l|}{ Affiliated department } \\
\hline ED & $81(78.6)$ \\
\hline Non-ED & $22(21.4)$ \\
\hline Clinical career (yr) & $5.0 \pm 5.0$ \\
\hline \multicolumn{2}{|l|}{ BLS or ACLS instructor } \\
\hline Yes & $13(12.6)$ \\
\hline No & $90(87.4)$ \\
\hline \multicolumn{2}{|l|}{ ACLS provider certification } \\
\hline Yes & $23(22.3)$ \\
\hline No & 80 (77.7) \\
\hline \multicolumn{2}{|c|}{ No. of CPR experiences (during last year) } \\
\hline 0 & $3(2.9)$ \\
\hline $1-5$ & $27(26.2)$ \\
\hline $6-10$ & $11(10.7)$ \\
\hline $11-15$ & $6(5.8)$ \\
\hline $16-20$ & $11(10.7)$ \\
\hline$>20$ & 45 (43.7) \\
\hline
\end{tabular}

Values are presented as number (\%) or mean \pm SD.

ED, emergency department; ACLS, advanced cardiac life support; CPR, cardiopulmonary resuscitation. accuracy rate was significantly higher among nurses $(P=0.02)$.

Table 3 shows environmental determinants associated with the estimated accuracy. The percentage of correct answers was significantly higher in video clips that were recorded at the bed side and in the caudal position than in the cephalic position $(\mathrm{P}<0.001)$. The estimated accuracy was significantly higher with female compressors $(P<0.001)$. The estimated accuracy was significantly lower in video clips with $4-5 \mathrm{~cm}$ or $5-6 \mathrm{~cm}$ compressions $(P<0.001)$.

The 5 factors associated with the estimated accuracy were selected and included for multivariate analysis. The results from logistic regression analysis of these factors are shown in Table 4. The adjusted odds ratio (AOR) of correct estimation was $1.50(95 \% \mathrm{Cl}$, 1.09 to 2.09; $P=0.01$ ) for nurses compared with physicians. The AOR was $8.19(95 \% \mathrm{Cl}, 5.40$ to $12.4 ; \mathrm{P}<0.001)$ for the caudal position compared with the cephalic position. The AORs were 0.68 0.03, and 0.06, respectively, for 3-4, 4-5, and 5-6 cm video clips compared with the $0-1 \mathrm{~cm}$ video clips.

Table 2. Examiner-determinants and estimation of accuracy

\begin{tabular}{|c|c|c|c|}
\hline \multirow{2}{*}{ Factor } & \multicolumn{2}{|c|}{ Rate of correct answers } & \multirow{2}{*}{ P-value } \\
\hline & Mean & $95 \% \mathrm{Cl}$ & \\
\hline Gender & & & 0.66 \\
\hline Male & 0.89 & $0.86-0.92$ & \\
\hline Female & 0.89 & $0.88-0.91$ & \\
\hline Age (yr) & & & 0.11 \\
\hline$<30$ & 0.90 & $0.88-0.92$ & \\
\hline$\geq 30$ & 0.88 & $0.85-0.90$ & \\
\hline Occupation & & & 0.02 \\
\hline Physician & 0.87 & $0.85-0.90$ & \\
\hline Nurse & 0.91 & $0.89-0.93$ & \\
\hline Emergency medical technician & 0.85 & $0.78-0.91$ & \\
\hline Affiliated department & & & 0.31 \\
\hline ED & 0.90 & $0.88-0.91$ & \\
\hline Non-ED & 0.88 & $0.84-0.91$ & \\
\hline Clinical career (yr) & & & 0.83 \\
\hline$<5$ & 0.89 & $0.87-0.91$ & \\
\hline$\geq 5$ & 0.89 & $0.87-0.92$ & \\
\hline BLS or ACLS instructor & & & 0.57 \\
\hline Yes & 0.88 & $0.84-0.92$ & \\
\hline No & 0.89 & $0.88-0.91$ & \\
\hline ACLS provider certification & & & 0.58 \\
\hline Yes & 0.88 & $0.85-0.92$ & \\
\hline No & 0.89 & $0.88-0.91$ & \\
\hline No. of CPR experiences (during last year) & & & 0.08 \\
\hline 0 & 0.92 & $0.66-1.18$ & \\
\hline $1-5$ & 0.92 & $0.90-0.95$ & \\
\hline $6-10$ & 0.86 & $0.80-0.92$ & \\
\hline $11-15$ & 0.85 & $0.76-0.95$ & \\
\hline $16-20$ & 0.89 & $0.86-0.93$ & \\
\hline$>20$ & 0.88 & $0.86-0.90$ & \\
\hline
\end{tabular}

$\mathrm{Cl}$, confidence interval; ED, emergency department; $\mathrm{ACLS}$, advanced cardiac life support; CPR, cardiopulmonary resuscitation. 
Table 3. Environmental determinants associated with estimation accuracy

\begin{tabular}{|c|c|c|c|}
\hline \multirow{2}{*}{ Factor } & \multicolumn{2}{|c|}{ Percentage of correct answers } & \multirow{2}{*}{ P-value } \\
\hline & Mean & 95\% Confidence interval & \\
\hline Camera position & & & $<0.001$ \\
\hline Cephalic & 0.77 & $0.74-0.79$ & \\
\hline Side & 0.96 & $0.94-0.97$ & \\
\hline Caudal & 0.96 & $0.95-0.97$ & \\
\hline Compressor gender & & & $<0.001$ \\
\hline Male & 0.86 & $0.84-0.88$ & \\
\hline Female & 0.91 & $0.90-0.93$ & \\
\hline Compression depth $(\mathrm{cm})$ & & & $<0.001$ \\
\hline$\geq 0 \&<1$ & 1.00 & $1.00-1.00$ & \\
\hline$\geq 1 \&<2$ & 1.00 & $1.00-1.00$ & \\
\hline$\geq 2 \&<3$ & 0.99 & $0.98-1.00$ & \\
\hline$\geq 3 \&<4$ & 0.92 & $0.89-0.96$ & \\
\hline$\geq 4 \&<5$ & 0.60 & $0.53-0.66$ & \\
\hline$\geq 5 \&<6$ & 0.70 & $0.66-0.74$ & \\
\hline$\geq 6 \&<7$ & 0.94 & $0.91-0.97$ & \\
\hline
\end{tabular}

\section{Sensitivity analysis}

We performed sensitivity analysis using different standards for the correct answers. For the ERC guideline-based analysis, the mean accuracy was $0.75(S D, 0.06)$. Multivariate analysis revealed statistically significant factors including camera position, compressor gender, and compression depth. For the strict standard, mean accuracy was only $0.40(S D, 0.11)$ and the significant factors were the same as the ERC guideline-based standard (Appendix Tables $1-6)$.

\section{DISCUSSION}

In this study, we investigated the accuracy of chest compression depth estimation. The accuracy rate was 0.89 (SD, 0.76), which was similar to previous research. ${ }^{11}$ However, when more strict criteria were applied, the estimated accuracy declined to 0.75 (SD, 0.40).

Although current guidelines have addressed the importance of mutual feedback, only a few have studied the effect. Lynch et al. ${ }^{11}$ found that examiner's estimation was not sufficient to determine providers' performance. Although multiple studies reported the importance of a feedback system, there was no research performed with health care providers in the field. ${ }^{12,13}$

Accuracy rates did not differ with clinical experience or gender, perhaps because measuring chest compressions is a very simple, low-tech procedure that does not require much experience. No difference in measuring skill was noted in previous studies either. ${ }^{14,15}$

In this study, the multivariate analysis showed nurses' superiority over physicians estimating compression depth, which may result from the tendency of nurses to follow protocols more strictly than physicians. Nurses tend to stand at a distance from chest
Table 4. Logistic regression predicting accurate estimation

\begin{tabular}{|c|c|c|c|c|}
\hline Factor & No. of clips & Odds ratio & $95 \% \mathrm{Cl}$ & P-value \\
\hline \multicolumn{5}{|l|}{ Examiner-determinants } \\
\hline \multicolumn{5}{|l|}{ Occupation } \\
\hline Physician & 1,050 & Reference & - & - \\
\hline Nurse & 1,400 & 1.50 & $1.09-2.09$ & 0.01 \\
\hline Emergency medical technician & 125 & 0.72 & $0.36-1.44$ & 0.35 \\
\hline \multicolumn{5}{|l|}{$\begin{array}{l}\text { No. of CPR experiences (during } \\
\text { last year) }\end{array}$} \\
\hline 0 & 75 & Reference & - & - \\
\hline $1-5$ & 675 & 1.22 & $0.43-3.41$ & 0.71 \\
\hline $6-10$ & 275 & 0.49 & $0.16-1.43$ & 0.19 \\
\hline $11-15$ & 150 & 0.48 & $0.15-1.52$ & 0.21 \\
\hline $16-20$ & 275 & 0.79 & $0.27-2.35$ & 0.67 \\
\hline$>20$ & 1,125 & 0.71 & $0.26-1.95$ & 0.50 \\
\hline \multicolumn{5}{|l|}{ Environment-determinants } \\
\hline \multicolumn{5}{|l|}{ Camera position } \\
\hline Cephalic & 927 & Reference & - & - \\
\hline Side & 618 & 0.73 & $0.36-1.43$ & 0.35 \\
\hline Caudal & 1,030 & 8.19 & $5.40-12.4$ & $<0.001$ \\
\hline \multicolumn{5}{|l|}{ Compressor gender } \\
\hline Female & 1,545 & Reference & - & - \\
\hline Male & 1,030 & 0.81 & $0.54-1.23$ & 0.32 \\
\hline \multicolumn{5}{|l|}{ Compression depth $(\mathrm{cm})$} \\
\hline$\geq 0 \&<1$ & 206 & Reference & - & - \\
\hline$\geq 1 \&<2$ & 515 & 1 & - & - \\
\hline$\geq 2 \&<3$ & 618 & 2.59 & $0.99-6.77$ & 0.05 \\
\hline$\geq 3 \&<4$ & 206 & 0.68 & $0.33-1.40$ & 0.30 \\
\hline$\geq 4 \&<5$ & 206 & 0.03 & $0.01-0.07$ & $<0.001$ \\
\hline$\geq 5 \&<6$ & 515 & 0.06 & $0.03-0.12$ & $<0.001$ \\
\hline$\geq 6 \&<7$ & 309 & 1 & - & - \\
\hline
\end{tabular}

$\mathrm{Cl}$, confidence interval; $\mathrm{CPR}$, cardiopulmonary resuscitation.

compression procedures, which may have given them more room to observe chest compression than physicians. However, factors not measured in this study, such as willingness to make an accurate estimation or professional experience, could have caused interactions among variables.

Camera position was highly correlated with the accuracy rate, indicating that one should stand at the side or foot of the patient to measure compression quality more accurately. In our study, accuracy rate dropped when the depth was near the target range, which emphasizes that the estimated accuracy in actual practice could be much lower than the result presented here. CPR team leaders should consider these factors while leading a CPR team. Another option could include a real-time feedback system for compression depth.

To improve the accuracy of estimation and feedback with precise information, applying new devices and measures is necessary. These measures include physiologic data such as end-tidal carbon dioxide levels, cerebral oximetry, and mechanically produced data such as compression depth by gyroscopes.

There are some major limitations to this study. First, study subjects did not fully represent the general population because sub- 
jects were enrolled from a single center. Second, watching an actual CPR process could be different from watching a video clip. Each individual used his or her own height and preferred position, which was standardized during the study. Third, the small number of participants could have influenced the negative outcomes. For example, EMT occupation showed an odds ratio of 0.70 without statistical significance. Because the number of enrolled EMTs was only 5 , more participants could have led to different results. Last, dividing compression depth into multiple scales would have complicated the determination of depth by examiners, which would have increased the inaccuracy of estimation.

In conclusions, the accuracy of chest compression depth estimation was $0.89(S D, 0.76)$ in this simulation model. The factors affecting accuracy were occupation, recording position, and compression depth itself.

\section{CONFLICT OF INTEREST}

No potential conflict of interest relevant to this article was reported.

\section{REFERENCES}

1. Koster RW, Baubin MA, Bossaert LL, et al. European Resuscitation Council Guidelines for Resuscitation 2010 Section 2: adult basic life support and use of automated external defibrillators. Resuscitation 2010;81:1277-92.

2. Berg RA, Hemphill $R$, Abella $B S$, et al. Part 5: adult basic life support: 2010 American Heart Association Guidelines for Cardiopulmonary Resuscitation and Emergency Cardiovascular Care. Circulation 2010;122(18 Suppl 3):S685-705.

3. American Heart Association. BLS for healthcare providers instructor manual. Dallas, TX: American Heart Association; 2010.

4. Deakin CD, Nolan JP, Soar J, et al. European Resuscitation Council Guidelines for Resuscitation 2010 Section 4: adult advanced life support. Resuscitation 2010;81:1305-52.
5. Abella BS, Edelson DP, Kim S, et al. CPR quality improvement during in-hospital cardiac arrest using a real-time audiovisual feedback system. Resuscitation 2007;73:54-61.

6. Spooner BB, Fallaha JF, Kocierz L, Smith CM, Smith SC, Perkins GD. An evaluation of objective feedback in basic life support (BLS) training. Resuscitation 2007;73:417-24.

7. Yeung J, Meeks R, Edelson D, Gao F, Soar J, Perkins GD. The use of CPR feedback/prompt devices during training and CPR performance: a systematic review. Resuscitation 2009;80:743-51.

8. Buleon C, Parienti JJ, Halbout L, et al. Improvement in chest compression quality using a feedback device (CPRmeter): a simulation randomized crossover study. Am J Emerg Med 2013; 31:1457-61.

9. Sainio $M$, Kamarainen $A$, Huhtala $H_{1}$ et al. Real-time audiovisual feedback system in a physician-staffed helicopter emergency medical service in Finland: the quality results and barriers to implementation. Scand J Trauma Resusc Emerg Med 2013;21:50.

10. Perkins GD, Augre $C$, Rogers $H$, Allan M, Thickett DR. CPREzy: an evaluation during simulated cardiac arrest on a hospital bed. Resuscitation 2005;64:103-8.

11. Lynch B, Einspruch EL, Nichol G, Aufderheide TP. Assessment of BLS skills: optimizing use of instructor and manikin measures. Resuscitation 2008;76:233-43.

12. Jiang $C$, Zhao $Y$, Chen $Z$, Chen $S$, Yang $X$. Improving cardiopulmonary resuscitation in the emergency department by realtime video recording and regular feedback learning. Resuscitation 2010;81:1664-9.

13. Akhtar N, Field RA, Davies RP, Perkins GD. Video-based CPR training: the importance of quality assurance. Resuscitation 2012;83:e158-9.

14. Ivin GF, Verrillo RT. Absolute estimation of line length and afterimage as a function of viewing distance. Sens Processes 1979;3:275-85.

15. Verrillo RT. Absolute estimation of line length in three age groups. J Gerontol 1981;36:625-7. 
Appendix Table 1. Examiner-determinants based on European Resuscitation Council guideline criteria

\begin{tabular}{|c|c|c|c|}
\hline \multirow{2}{*}{ Factor } & \multicolumn{2}{|c|}{ Percentage of correct answers } & \multirow{2}{*}{ P-value } \\
\hline & Mean & $95 \% \mathrm{Cl}$ & \\
\hline Gender & & & 0.94 \\
\hline Male & 0.75 & $0.73-0.78$ & \\
\hline Female & 0.75 & $0.74-0.77$ & \\
\hline Age (yr) & & & 0.26 \\
\hline$<30$ & 0.76 & $0.74-0.77$ & \\
\hline$\geq 30$ & 0.74 & $0.72-0.76$ & \\
\hline Occupation & & & 0.81 \\
\hline Physician & 0.75 & $0.73-0.77$ & \\
\hline Nurse & 0.76 & $0.74-0.77$ & \\
\hline Emergency medical technician & 0.74 & $0.70-0.79$ & \\
\hline Affiliated department & & & 0.72 \\
\hline ED & 0.75 & $0.74-0.76$ & \\
\hline Non-ED & 0.76 & $0.73-0.78$ & \\
\hline Clinical career (yr) & & & 0.96 \\
\hline$<5$ & 0.75 & $0.74-0.77$ & \\
\hline$\geq 5$ & 0.75 & $0.73-0.77$ & \\
\hline Instructor certification & & & 0.39 \\
\hline Yes & 0.74 & $0.70-0.77$ & \\
\hline No & 0.75 & $0.74-0.77$ & \\
\hline ACLS certification & & & 0.49 \\
\hline Yes & 0.74 & $0.71-0.78$ & \\
\hline No & 0.75 & $0.74-0.77$ & \\
\hline $\begin{array}{l}\text { No. of CPR experience (during } \\
\text { last year) }\end{array}$ & & & 0.57 \\
\hline 0 & 0.77 & $0.54-1.00$ & \\
\hline $1-5$ & 0.77 & $0.75-0.79$ & \\
\hline $6-10$ & 0.73 & $0.69-0.77$ & \\
\hline $11-15$ & 0.73 & $0.66-0.80$ & \\
\hline $16-20$ & 0.76 & $0.72-0.80$ & \\
\hline$>20$ & 0.75 & $0.73-0.77$ & \\
\hline
\end{tabular}

$\mathrm{Cl}$, confidence interval; ED, emergency department; $\mathrm{ACLS}$, advanced cardiac life support; CPR, cardiopulmonary resuscitation.

Appendix Table 2. Environment-determinants based on European Resuscitation Council guideline standard

\begin{tabular}{|c|c|c|c|}
\hline \multirow{2}{*}{ Factor } & \multicolumn{2}{|c|}{ Percentage of correct answers } & \multirow{2}{*}{ P-value } \\
\hline & Mean & $95 \% \mathrm{Cl}$ & \\
\hline Camera position & & & $<0.001$ \\
\hline Cephalic & 0.64 & $0.61-0.67$ & \\
\hline Side & 0.80 & $0.77-0.83$ & \\
\hline Caudal & 0.83 & $0.81-0.85$ & \\
\hline Compressor gender & & & 0.001 \\
\hline Male & 0.79 & $0.76-0.81$ & \\
\hline Female & 0.73 & $0.71-0.75$ & \\
\hline Compression depth $(\mathrm{cm})$ & & & $<0.001$ \\
\hline $0-1$ & 1.00 & $1.00-1.00$ & \\
\hline $1-2$ & 1.00 & $1.00-1.00$ & \\
\hline $2-3$ & 1.00 & $1.00-1.00$ & \\
\hline $3-4$ & 0.99 & $0.98-1.00$ & \\
\hline $4-5$ & 0.70 & $0.64-0.77$ & \\
\hline $5-6$ & 0.28 & $0.24-0.32$ & \\
\hline $6-7$ & 0.34 & $0.28-0.39$ & \\
\hline
\end{tabular}

$\mathrm{Cl}$, confidence interval.
Appendix Table 3. Logistic regression based on European Resuscitation Council guideline criteria

\begin{tabular}{|c|c|c|c|c|}
\hline Factor & No. of clip & Odds ratio & $95 \% \mathrm{Cl}$ & P-value \\
\hline \multicolumn{5}{|l|}{ Occupation } \\
\hline Physician & 1,050 & Reference & - & - \\
\hline Nurse & 1,400 & 1.01 & $0.75-1.36$ & 0.94 \\
\hline Emergency medical technician & 125 & 0.95 & $0.48-1.85$ & 0.87 \\
\hline \multicolumn{5}{|l|}{$\begin{array}{l}\text { No. of CPR experience (during } \\
\text { last year) }\end{array}$} \\
\hline 0 & 75 & Reference & - & - \\
\hline $1-5$ & 675 & 0.92 & $0.40-2.09$ & 0.84 \\
\hline $6-10$ & 275 & 0.60 & $0.24-1.47$ & 0.26 \\
\hline $11-15$ & 150 & 0.62 & $0.23-1.65$ & 0.34 \\
\hline $16-20$ & 275 & 0.86 & $0.35-2.08$ & 0.74 \\
\hline$>20$ & 1,125 & 0.75 & $0.33-1.70$ & 0.49 \\
\hline \multicolumn{5}{|l|}{ Camera position } \\
\hline Cephalic & 927 & Reference & - & - \\
\hline Side & 618 & 3.64 & $2.06-6.45$ & $<0.001$ \\
\hline Caudal & 1,030 & 0.89 & $0.64-1.25$ & 0.50 \\
\hline \multicolumn{5}{|l|}{ Compressor gender } \\
\hline Female & 1,545 & Reference & & \\
\hline Male & 1,030 & 0.39 & $0.26-0.60$ & $<0.001$ \\
\hline \multicolumn{5}{|l|}{ Compression depth (cm) } \\
\hline $0-1$ & 206 & Reference & - & - \\
\hline $1-2$ & 515 & 1.00 & - & - \\
\hline $2-3$ & 618 & 1.00 & - & - \\
\hline $3-4$ & 206 & 235 & $55.5-995$ & $<0.001$ \\
\hline $4-5$ & 206 & 23.0 & $11.1-47.7$ & $<0.001$ \\
\hline $5-6$ & 515 & 1.72 & $1.01-2.90$ & 0.05 \\
\hline $6-7$ & 309 & 1.00 & - & - \\
\hline
\end{tabular}

$\mathrm{Cl}$, confidence interval; $\mathrm{CPR}$, cardiopulmonary resuscitation. 
Appendix Table 4. Examiner-determinants based on strict criteria

\begin{tabular}{|c|c|c|c|}
\hline \multirow{2}{*}{ Factor } & \multicolumn{2}{|c|}{ Percentage of correct answers } & \multirow{2}{*}{ P-value } \\
\hline & Mean & $95 \% \mathrm{Cl}$ & \\
\hline \multicolumn{3}{|l|}{ Gender } & 0.88 \\
\hline Male & 0.41 & $0.36-0.45$ & \\
\hline Female & 0.40 & $0.38-0.43$ & \\
\hline \multicolumn{3}{|l|}{ Age (yr) } & 0.55 \\
\hline$<30$ & 0.41 & $0.38-0.44$ & \\
\hline$\geq 30$ & 0.40 & $0.36-0.43$ & \\
\hline \multicolumn{3}{|l|}{ Occupation } & 0.30 \\
\hline Physician & 0.39 & $0.36-0.43$ & \\
\hline Nurse & 0.42 & $0.39-0.45$ & \\
\hline Emergency medical technician & 0.35 & $0.16-0.55$ & \\
\hline \multicolumn{3}{|l|}{ Affiliated department } & 0.66 \\
\hline ED & 0.41 & $0.38-0.43$ & \\
\hline Non-ED & 0.39 & $0.35-0.44$ & \\
\hline \multicolumn{3}{|l|}{ Clinical career (yr) } & 0.53 \\
\hline$<5$ & 0.40 & $0.37-0.43$ & \\
\hline$\geq 5$ & 0.41 & $0.38-0.45$ & \\
\hline \multicolumn{3}{|l|}{ Instructor certification } & 0.33 \\
\hline Yes & 0.38 & $0.29-0.46$ & \\
\hline No & 0.41 & $0.39-0.43$ & \\
\hline \multicolumn{3}{|l|}{ ACLS certification } & 0.65 \\
\hline Yes & 0.39 & $0.34-0.45$ & \\
\hline No & 0.41 & $0.38-0.43$ & \\
\hline \multicolumn{3}{|c|}{ No. of CPR experience (during last year) } & 0.03 \\
\hline 0 & 0.45 & $0.05-0.85$ & \\
\hline $1-5$ & 0.46 & $0.42-0.49$ & \\
\hline $6-10$ & 0.35 & $0.29-0.42$ & \\
\hline $11-15$ & 0.33 & $0.23-0.44$ & \\
\hline $16-20$ & 0.36 & $0.29-0.44$ & \\
\hline$>20$ & 0.40 & $0.37-0.44$ & \\
\hline
\end{tabular}

$\mathrm{Cl}$, confidence interval; $E D$, emergency department; $A C L S$, advanced cardiac life support; CPR, cardiopulmonary resuscitation.

Appendix Table 5. Environment-determinants based on strict criteria

\begin{tabular}{|c|c|c|c|}
\hline \multirow{2}{*}{ Factor } & \multicolumn{2}{|c|}{ Percentage of correct answers } & \multirow{2}{*}{ P-value } \\
\hline & Mean & $95 \% \mathrm{Cl}$ & \\
\hline Camera position & & & $<0.001$ \\
\hline Cephalic & 0.23 & $0.20-0.26$ & \\
\hline Side & 0.53 & $0.49-0.57$ & \\
\hline Caudal & 0.49 & $0.46-0.52$ & \\
\hline Compressor gender & & & 0.005 \\
\hline Male & 0.37 & $0.34-0.40$ & \\
\hline Female & 0.43 & $0.40-0.45$ & \\
\hline Compression depth (cm) & & & $<0.001$ \\
\hline $0-1$ & 0.89 & $0.84-0.93$ & \\
\hline $1-2$ & 0.43 & $0.39-0.48$ & \\
\hline $2-3$ & 0.41 & $0.38-0.45$ & \\
\hline $3-4$ & 0.33 & $0.26-0.39$ & \\
\hline $4-5$ & 0.30 & $0.24-0.36$ & \\
\hline $5-6$ & 0.28 & $0.24-0.32$ & \\
\hline $6-7$ & 0.34 & $0.28-0.39$ & \\
\hline
\end{tabular}

$\mathrm{Cl}$, confidence interval.
Appendix Table 6. Logistic regression based on strict criteria

\begin{tabular}{lrccc}
\hline Factor & No. of clip & Odds ratio & $95 \% \mathrm{Cl}$ & P-value \\
\hline Occupation & & & & \\
$\quad$ Physician & 1,050 & Reference & - & - \\
Nurse & 1,400 & 1.04 & $0.87-1.25$ & 0.67 \\
$\quad$ Emergency medical & 125 & 0.77 & $0.50-1.19$ & 0.24 \\
Number of CPR experience & & & & \\
$\quad$ (during last year) & & & & \\
0 & 75 & Reference & - & - \\
$1-5$ & 675 & 1.02 & $0.61-1.72$ & 0.93 \\
$6-10$ & 275 & 0.63 & $0.36-1.10$ & 0.11 \\
$11-15$ & 150 & 0.57 & $0.30-1.06$ & 0.08 \\
$16-20$ & 275 & 0.66 & $0.38-1.16$ & 0.15 \\
$>20$ & 1,125 & 0.82 & $0.49-1.38$ & 0.46 \\
Camera position & & & & \\
Cephalic & 927 & Reference & - & - \\
Side & 618 & 2.62 & $2.01-3.42$ & $<0.001$ \\
Caudal & 1,030 & 2.49 & $2.00-3.09$ & $<0.001$ \\
Compressor gender & & & & \\
Female & 1,545 & Reference & - & - \\
Male & 1,030 & 0.69 & $0.56-0.85$ & 0.001 \\
Compression depth (cm) & & & & \\
0-1 & 206 & Reference & - & - \\
$1-2$ & 515 & 0.13 & $0.08-0.21$ & $<0.001$ \\
$2-3$ & 618 & 0.10 & $0.06-0.16$ & $<0.001$ \\
3-4 & 206 & 0.07 & $0.04-0.13$ & $<0.001$ \\
$4-5$ & 206 & 0.09 & $0.06-0.17$ & $<0.001$ \\
$5-6$ & 515 & 0.07 & $0.04-0.12$ & $<0.001$ \\
$6-7$ & 309 & 0.08 & $0.05-0.13$ & $<0.001$ \\
\hline
\end{tabular}

$\mathrm{Cl}$, confidence interval; $\mathrm{CPR}$, cardiopulmonary resuscitation. 